\title{
Induced ferromagnetism due to superconductivity in superconductor-ferromagnet structures
}

\author{
F. S. Bergeret, ${ }^{1,2}$ A. F. Volkov, ${ }^{1,3}$ and K. B. Efetov ${ }^{1,4}$ \\ ${ }^{1}$ Theoretische Physik III, Ruhr-Universität Bochum, D-44780 Bochum, Germany \\ ${ }^{2}$ Laboratorio de Física de Sistema Pequeños y Nanotecnología, CSIC, 28006 Madrid, Spain \\ ${ }^{3}$ Institute of Radioengineering and Electronics of the Russian Academy of Sciences, 103907 Moscow, Russia \\ ${ }^{4}$ L. D. Landau Institute for Theoretical Physics, 117940 Moscow, Russia
}

(Received 11 September 2003; revised manuscript received 2 March 2004; published 27 May 2004)

\begin{abstract}
We consider a superconductor-ferromagnet (S/F) structure and assume that above the superconducting transition temperature $T_{c}$ the magnetic moment exists only in $\mathrm{F}$. In a simple model of the ferromagnet (the exchange field is of the ferromagnetic type for all energies) we show by an explicit calculation that below $T_{c}$ the magnetic moment may penetrate the superconductor. In this model its direction in $\mathrm{S}$ is opposite to the magnetization of free electrons in the ferromagnet. The magnetization spreads over a large distance which is of the order of the superconducting coherence length $\xi_{S}$ and can much exceed the ferromagnet film thickness. At the same time the magnetic moment in the ferromagnet is reduced. This inverse proximity effect may explain the reduction in magnetization observed in recent experiments and may lead to a strong interaction between the ferromagnetic layers in F/S/F structures.
\end{abstract}

DOI: 10.1103/PhysRevB.69.174504

PACS number(s): 74.45.+c, 74.78.Fk

Penetration of the superconducting condensate into a normal metal in the superconductor $(\mathrm{S})$-normal metal $(\mathrm{N})$ heterostructures is a well established proximity effect. The latter is a long-range effect because the amplitude of the condensate decays in the normal metal very slowly with a characteristic length $\xi_{N}$ which in the dirty limit is equal to $\xi_{N}$ $=\sqrt{D_{N} / 2 \pi T}\left(D_{N}\right.$ is the diffusion coefficient in the normal metal and $T$ is the temperature). At low temperatures this length can be very large. At the same time, the order parameter $\Delta$ in the superconductor near the $\mathrm{S} / \mathrm{N}$ interface is suppressed. The magnitude of the suppression depends on the parameters characterizing the system such as the $\mathrm{S} / \mathrm{N}$ interface transparency, the thickness of the $\mathrm{S}$ and $\mathrm{N}$ layers, etc. ${ }^{1}$ The proximity effect arises also in superconductorferromagnet $(\mathrm{S} / \mathrm{F})$ structures. While the superconducting condensate consists of paired electrons with opposite spins, the exchange field $J$ in the ferromagnet tends to align them and break the Cooper pairs. The penetration length $\xi_{F}$ of the condensate into the ferromagnet is usually much smaller than $\xi_{N}$ and in the dirty limit is equal to $\xi_{F}=\sqrt{D_{F} / J}$ ( $D_{F}$ is the diffusion coefficient in the ferromagnet). Since the exchange energy $J$ is much larger than $T$, we come to the inequality $\xi_{F} \ll \xi_{N}$ (in the clean limit when $\tau J \gg 1$, the penetration length $\xi_{F}$ is determined by the mean-free-path $l=v_{F} \tau$, where $v_{F}$ is the Fermi velocity ${ }^{2-4}$ ). A strong exchange field suppresses also the superconducting order parameter $\Delta$ in the superconductor.

The situation changes when the magnetization $\mathbf{M}$ in the ferromagnet is not homogeneous. In this case a triplet component of the condensate with a nonzero spin projection arises and penetrates into the $\mathrm{F}$ region over a long distance of the order $\sqrt{D_{F} / 2 \pi T}$ (see Refs. 5 and 6). The effect of the penetration of the superconducting condensate into the ferromagnet and the suppression of the superconductivity (a decrease of the critical temperature $T_{c}$ of the superconducting transition) in S/F structures, i.e., the proximity effect, have been the subject of many works during the last decades (see for example Refs. $7-9$ and the review ${ }^{10}$ for more references).
So, the penetration of the superconductivity into the normal metal or ferromagnet is by now a very well studied phenomenon. However, one can ask the same question about the ferromagnetism: Can the ferromagnetic order penetrate the normal metal or superconductor over long distances? Surprisingly, this question has hardly been addressed. Some indications of the effect can be found in numerical works. ${ }^{9,11}$ In these works only the density of states for each spin direction as a function of the energy was presented, however, the magnetization was not calculated. In addition the induced magnetization (magnetization leakage) was calculated in Ref. 12. However, the results obtained in the latter paper generally speaking differ drastically from ours. They found a magnetization leakage that is the magnetic moment of free electrons $M_{e}$ spreads into the $\mathrm{S}$ region over a distance of the order $\xi_{S}$ changing its sign at some distance from the S/F interface.

We consider a simple model assuming a mean-field approximation for the ferromagnet and superconductor. The mean-field order parameter in $\mathrm{S}$ is the energy gap $\Delta$, and in $\mathrm{F}$ it is the exchange field $J$ which is assumed to be of the ferromagnetic type and small compared with the Fermi energy. In different limiting cases where analytical formulas can be obtained we find completely different behavior: For temperatures below $T_{c}$, the magnetization of free electrons in the $\mathrm{F}$ layer $M_{e}$ decreases and the induced magnetization in the $S$ region is negative (that is, the magnetization variation has the same negative sign in both regions). Our analytical considerations show that no change of sign of the induced magnetization takes place. This behavior is in agreement with the reduction of magnetization observed in the experiments of Refs. 13 and 14 and can be explained by the simple physical picture we present below.

In the case of $\mathrm{F} / \mathrm{N}$ systems the ferromagnetic ordering penetrates over short distances since the exchange interaction is local. In this paper we show that the situation may be different for $\mathrm{S} / \mathrm{F}$ structures and present arguments that the magnetic moment can penetrate into the superconductor over 


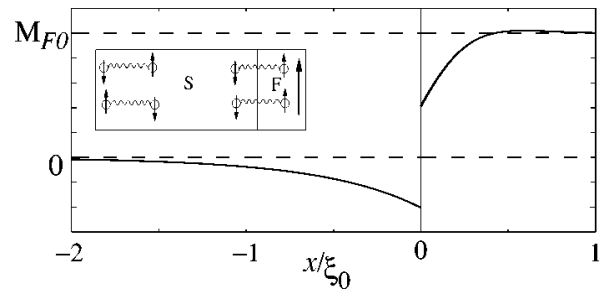

FIG. 1. Spatial dependence of the magnetization in the whole system. Here $\gamma_{F} / \gamma_{S}=0.5, \bar{\gamma}_{F}=\gamma_{F} / \xi_{0}=0.1\left(\xi_{0}=\sqrt{D_{S} / 2 T_{c}}\right), J / T_{c}$ $=15$, and $d_{F} / \xi_{0}=1$. Inset: Schematic view of the inverse proximity effect in a $\mathrm{S} / \mathrm{F}$ system (for discussion see text).

long distances of the order of the superconducting coherence length. This effect can be called the inverse proximity effect. The reason why the magnetic moment aligned in the direction opposite to the magnetization in the $\mathrm{F}$ film, $\mathbf{M}_{e}$, penetrates the superconductor can rather easily be understood qualitatively. This effect is due to the fact that the Cooper pairs have a large size of the order of $\xi_{S} \cong \sqrt{D_{S} / 2 \pi T_{c}}$. Suppose that the $\mathrm{F}$ layer is thin (see inset of Fig. 1) and let us assume that the Cooper pairs are rigid objects consisting of electrons with opposite spins, such that the total magnetic moment of a pair is equal to zero. Of course, the exchange field should not be very strong, otherwise the pairs would break down. It is clear from this simple picture that pairs located entirely in the superconductor cannot contribute to the magnetic moment of the superconductor because their magnetic moment is simply zero. Nevertheless, some pairs are located in space in a more complicated manner: one of the electrons of the pair is in the superconductor, while the other moves in the ferromagnet. These are those pairs that create the magnetic moment in the superconductor. The direction along the magnetic moment $\mathbf{M}_{e}$ in the ferromagnet is preferable for the electron located in the ferromagnet and this makes the spin of the other electron of the pair to be antiparallel to $\mathbf{M}_{e}$. This means that all such pairs equally contribute to the magnetic moment in the bulk of the superconductor. As a result, a ferromagnetic order is created in the superconductor and the direction of the magnetic moment in this region is opposite to the direction of the magnetic moment $\mathbf{M}_{e}$ in the ferromagnet. Moreover, the induced magnetic moment penetrates over the size of the Cooper pairs $\xi_{S}$. From this point of view it is difficult to understand the numerical results of Ref. 12 where the induced magnetization in the $\mathrm{S}$ region near the $S / F$ interface has the same sign as in the ferromagnet. The magnetic moment in the ferromagnet is decreased because the density of states in $\mathrm{F}$ is reduced due to the proximity effect. This occurs in a way similar to a suppression of the Pauli paramagnetism in superconductors (the exchange field plays the role of a strong magnetic field acting on spins). At the same time the concentrations of free electrons with spin up and down in F remain unchanged; when we are saying about the penetration of Cooper pairs into the ferromagnet $\mathrm{F}$, we mean that superconducting correlations are established in $\mathrm{F}$ due to the proximity effect. Having presented the qualitative picture, we calculate now the magnetization variation due to free electrons (the conduction-band electrons),

$$
\delta M_{e}=\mu_{B} \delta N_{M},
$$

below $T_{c}$ in both layers of the $\mathrm{S} / \mathrm{F}$ system shown in the inset of Fig. 1. Here $\mu_{B}$ is an effective Bohr magneton. We assume that the magnetic moment $\mathbf{M}_{e}$ is parallel to the interface as it takes place in the experiment ${ }^{13,14}$ and is homogeneous in the F layer. As we have found previously, Ref. 15, in this case only the singlet component and the triplet one with the zero-spin projection on the direction of $\mathbf{M}_{e}$ exist in the system. Both components penetrate into the ferromagnet over the short distance $\xi_{F}$. If the $\mathrm{S} / \mathrm{F}$ interface transparency is low or the conductivity of the $\mathrm{S}$ film is much higher than the conductivity of the F film, the suppression of the order parameter $\Delta$ is not essential and the superconducting properties remain almost unchanged. The quantity $\delta N_{M}$ can be expressed in terms of the quasiclassical normal Green function $\hat{g}$ :

$$
\delta N_{M}=\sum_{p}\left(\left\langle c_{p \uparrow}^{\dagger} c_{p \uparrow}-c_{p \downarrow}^{\dagger} c_{p \downarrow}\right\rangle\right)=-i \pi \nu T \sum_{\omega=-\infty}^{\omega=+\infty} \operatorname{Tr}\left(\hat{\sigma}_{3} \hat{g}\right),
$$

where $\nu=p_{F} m /\left(2 \pi^{2}\right)$ is the density of states at the Fermi level, $\hat{\sigma}_{3}$ is the third Pauli matrix, and $\omega=\pi T(2 n+1)$ is the Matsubara frequency. The normal Green function $\hat{g}$ is a matrix in the spin space. In the considered case of a uniform magnetization it has the form $\hat{g}=g_{0} \cdot \hat{\sigma}_{0}+g_{3} \cdot \hat{\sigma}_{3}$. This matrix is related to the Gor'kov anomalous matrix Green function $\hat{f}$ via the normalization condition

$$
\hat{g}^{2}-\hat{f}^{2}=1
$$

The matrix $\hat{f}$ describes the superconducting condensate. In order to visualize how our results are obtained, we consider first the simplest case when the condensate function $\hat{f}$ is small in F and is close to its bulk value in the superconductor. We analyze the dirty case when the Usadel equation can be applied. This means that s-wave superconductors are considered. These are described by the standard BCS Hamiltonian with account for the exchange field (in the ferromagnet) acting on the spins of the free electrons. We write the Hamiltonian in the form

$$
\hat{H}=\hat{H}_{o}+\hat{H}_{S}+\hat{H}_{F} .
$$

Here $\hat{H}_{o}$ is the one-particle Hamiltonian which includes the impurity scattering term $U_{i m p}$,

$$
\hat{H}_{o}=\sum_{\{p, s\}}\left\{a_{s p}^{+}\left[\xi_{p} \delta_{p p^{\prime}}+U_{i m p} \delta_{s s^{\prime}}\right] a_{s^{\prime} p^{\prime}}\right\}
$$

where $\xi_{p}=p^{2} / 2 m-\epsilon_{F}$ is the kinetic energy counted from the Fermi energy $\epsilon_{F}$. The second term in Eq. (4) is the standard BCS Hamiltonian for the superconductor written in the mean-field approximation,

$$
\hat{H}_{S}=-\sum_{\{p, s\}}\left\{\Delta a \frac{+}{s p} a_{s p}^{+}+\text {c.c. }\right\}
$$


where $\bar{s}=-s(s= \pm 1)$ and $\bar{p}=-p$. The last term in Eq. (4) describes the ferromagnetic interaction in $\mathrm{F}$. We accept the simplest form of this part of the Hamiltonian (the mean-field approximation),

$$
\hat{H}_{F}=-\sum_{\{p, s\}} J\left\{a_{s p}^{+} \mathbf{n} \cdot \boldsymbol{\sigma}_{s s^{\prime}} a_{s^{\prime} p^{\prime}}\right\},
$$

where the exchange energy is assumed to be positive for all energies (the ferromagnetic type of interaction), $n$ is the unit vector parallel to the magnetization of the ferromagnet. The magnetization $M$ of the ferromagnet is proportional to the exchange energy $J$. If the contribution of free electrons strongly dominates (an itinerant ferromagnet), one has $M$ $\cong M_{e}$.

If the polarization of the conduction electrons is due to the interaction with localized magnetic moments, the Hamiltonian $\hat{H}_{F}$ may be written in the form ${ }^{16,17,19}$

$$
\hat{H}_{F}=-J_{1} \sum_{\{p, s\}}\left\{a_{s p}^{+} \mathbf{S} \cdot \sigma_{s s^{\prime}} a_{s^{\prime} p^{\prime}}\right\},
$$

where $S=\sum_{a} S_{a} \delta\left(r-r_{a}\right), S_{a}$ is the spin of a particular ion. A constant $J_{1}$ is related to $J$ via the equation: $J=J_{1} n_{M} S_{0}$, where $n_{M}$ is the concentration of magnetic ions and $S_{0}$ is a maximum value of $S_{a}$ (we consider these spins as classical vectors; see Ref. 16). In this case the magnetization is a sum: $\mathbf{M}=\mathbf{M}_{l o c}+\mathbf{M}_{e}$, and the magnetization $\mathbf{M}_{e}$ may be aligned parallel $\left(J_{1}>0\right.$, the ferromagnetic type of the exchange field) to $\mathbf{M}$ or antiparallel $\left(J_{1}<0\right.$, the antiferromagnetic type of the exchange field). In the following we will assume a ferromagnetic exchange interaction $\left(\mathbf{M}_{e}\right.$ and $\mathbf{M}$ are oriented in the same direction). The case of antiferromagnetic coupling will be briefly discussed below. In principle one can add to Eq. (8) the term $\Sigma_{\{a, b\}}\left\{\mathbf{S}_{a}^{*} \mathbf{S}_{b}\right\}$ which describes a direct interaction between localized magnetic moments, ${ }^{17,19}$ but this term does not affect the final results.

Starting from the Hamiltonian (4) and using a standard approach, ${ }^{18}$ one can derive the Usadel equation. In the case of a low S/F interface transparency this equation can be linearized. Then, the function $\hat{f}$ is obtained from the linearized Usadel equation (see e.g. Ref. 15)

$$
\partial_{x x}^{2} f_{ \pm}-\kappa_{ \pm}^{2} f_{ \pm}=0 \quad \text { in the } \mathrm{F} \text { layer }
$$

and

$$
\partial_{x x}^{2} \delta \hat{f}_{S}-\kappa_{S}^{2} \delta \hat{f}_{S}=\mathcal{K}(x) \hat{\sigma}_{3} \quad \text { in the } \mathrm{S} \text { layer. }
$$

Here $\kappa_{ \pm}^{2}=2(|\omega| \mp i J \operatorname{sgn} \omega) / D_{F}, \quad \kappa_{S}^{2}=2 \sqrt{\omega^{2}+\Delta^{2}} / D_{S} \quad$ and $\delta \hat{f}_{S}$ is a deviation of the function $\hat{f}_{S}$ from its bulk (BCS) value $f_{B C S}$, i.e., $\delta \hat{f}_{S}=\hat{f}_{S}-\hat{f}_{B C S}, \hat{f}_{B C S}=f_{B C S} \cdot \hat{\sigma}_{3}$. The functions $f_{F \pm}$ are the elements $(1,1)$ and $(2,2)$ of the matrix $\hat{f}_{F}$. The function $\mathcal{K}(x)$ contains the correction $\delta \Delta(x)$ to the order parameter $\Delta$. This term is not relevant in our calculations since only the component of $\hat{f}$ proportional to $\hat{\sigma}_{0}$ contributes to the magnetization [see below, Eq. (17)]. Equations (9) and (10) should be complemented by the boundary conditions that can be written for small $\hat{f}_{S, F}$ as

$$
\begin{gathered}
\partial_{x} \delta \hat{f}_{S}=\left(g_{B C S}^{2} \cdot \hat{f}_{F}-g_{B C S} f_{B C S} \operatorname{sgn} \omega \cdot \hat{\sigma}_{3}\right) / \gamma_{S}, \\
\partial_{x} \hat{f}_{F}=-\left(1 / \gamma_{F}\right) \hat{f}_{S},
\end{gathered}
$$

where $\gamma_{S, F}=R_{b} \sigma_{S, F}, R_{b}$ is the $\mathrm{S} / \mathrm{F}$ interface resistance per unit area, $\sigma_{S, F}$ is the conductivity of the $\mathrm{S}$ or $\mathrm{F}$ region, and $\hat{g}_{B C S}=g_{B C S} \cdot \hat{\sigma}_{0}$. The BCS functions have the well-known form (see, for example, Ref. 18)

$$
g_{B C S}=\omega / \sqrt{\omega^{2}+\Delta^{2}}, \quad f_{B C S}=\Delta / i \sqrt{\omega^{2}+\Delta^{2}} .
$$

The matrix function of the superconducting condensate $\hat{f}$ can be represented in the form

$$
\hat{f}=f_{3} \hat{\sigma}_{3}+f_{0} \hat{\sigma}_{0}
$$

for both regions. The component $f_{3}$ describes the singlet condensate, whereas $f_{0}$ stands for the triplet component with the zero projection of the total spin of the pair on the direction of the magnetic moment $\mathbf{M}$. These functions are related to $f_{ \pm}$through: $f_{0,3}(x)=(1 / 2)\left[f_{+}(x) \pm f_{-}(x)\right]$. The other components of the triplet condensate arise only if $\mathbf{M}$ in the ferromagnet is inhomogeneous.

Solving Eqs. (9) and (10) with the boundary conditions, Eqs. (11) and (12), we find easily

$$
\begin{gathered}
f_{F \pm}(x)=b_{ \pm} \exp \left(-\kappa_{ \pm} x\right), \\
f_{S 0}(x)=-a_{0} \exp \left(\kappa_{S} x\right) .
\end{gathered}
$$

Here $b_{ \pm}= \pm f_{B C S} /\left(\gamma_{F} \kappa_{ \pm}\right)$and $a_{0}=g_{B C S}^{2} f_{F 0}(0) /\left(\gamma_{S} \kappa_{S}\right)$. As follows from Eqs. (15) and (16) the functions $f_{F \pm}$ and $f_{S 0}(x)$ are small provided that $R_{F} / R_{b} \ll 1$ and $\left(R_{F} / R_{b}\right)\left(R_{S} / R_{b}\right)$ $\ll 1$, where $R_{F, S}=\xi_{F, S} / \sigma_{F, S}$ are the resistances (per unit area) of the $\mathrm{F}(\mathrm{S})$ of lengths $\xi_{F, S}$. In order to calculate the magnetization we have to find the function $g_{3}=\operatorname{Tr}\left(\hat{\sigma}_{3} \hat{g} / 2\right)$ [see Eq. (2)]. The latter is related to the functions $f_{0,3}$ in the $\mathrm{F}$ and $\mathrm{S}$ region through the normalization condition, Eq. (3), and is given by

$$
g_{F 3}=f_{F 0} f_{F 3} \operatorname{sgn} \omega, \quad g_{S 3}=f_{B C S} \delta f_{S 0} / g_{B S C} .
$$

As it has been discussed in Ref. 15, the functions $f_{S 0}$ and $f_{F 0}$ corresponding to the triplet component of the condensate are odd functions of $\omega$ while the singlet components $f_{B C S}$ and $f_{F 3}$ are even functions. Thus, according to Eqs. (17) the functions $g_{F 3}$ and $g_{S 3}$ are even functions of $\omega\left(g_{B S C}\right.$ is odd in $\omega)$. This means that the sum over the frequencies in Eq. (2) is not zero and the proximity effect leads to a change $\delta M_{e}$ of the magnetization in both $\mathrm{F}$ and $\mathrm{S}$ layers (above $T_{c}$ the magnetization in $\mathrm{S}$ is zero).

After the qualitative discussion we have come to the conclusion that the net magnetization due to the inverse proximity effect must be negative. The explicit calculation based on Eqs. (2) and (15)-(17) confirms this result which is shown in Fig. 1 for some values of the parameters. We see that $\delta M_{e}$ is negative, i.e., the magnetization of the ferromagnet is reduced and the superconductor acquires a finite magnetization in the opposite direction. The change of the magnetization $\delta M(x)$ extends over the length $\kappa_{s}^{-1}$, which may be much 
larger than the thickness of the F layer. This effect is another manifestation of the existence of the triplet component of $\hat{f}$.

The inverse proximity effect considered here may be relevant for several experiments on the measurements of the magnetization in the S/F structures. ${ }^{13,14}$ In these experiments it was found that the magnetization started to decrease when crossing the superconducting critical temperature $T_{c}$ from above. The authors of these experiments compared the data with the theoretical results of Refs. 19 and 20 that were obtained under the assumption that the ferromagnetic order in the $\mathrm{F}$ thin layer might be modified due to the proximity effect leading to the so-called cryptoferromagnetic state. In these works only the contribution of localized moments to the magnetization was taken into account. Our calculations show that the conduction electrons can give an additional contribution. The inverse proximity effect leads to an additional reduction of the magnetization $M_{e}$ and may serve as an alternative explanation for the reduction of the magnetization observed experimentally. ${ }^{13,14}$

Let us analyze now an interesting case that may be relevant to the experimental situation of Ref. 14. We assume that the thickness of the F layer $d_{F}$ is small compared to $\xi_{F}$ and that the Green functions $g_{S}$ and $f_{S}$ are close to the bulk values $g_{B S C}$ and $f_{B C S}$. The latter assumptions are valid if the coefficient $\gamma_{F} / \gamma_{S}=\sigma_{F} / \sigma_{S}$ is small enough. In this case all functions in the $\mathrm{F}$ region are not necessarily small but they are almost constant in space. Therefore we can average the exact Usadel equation over $x$ taking into account exact boundary conditions. Proceeding in this way, we get for the diagonal elements $g_{ \pm}$and $f_{ \pm}$of the matrices $\hat{g}$ and $\hat{f}$,

$$
g_{F \pm}=\tilde{\omega}_{ \pm} / \zeta_{\omega \pm}, \quad f_{F \pm}= \pm \epsilon_{b F} f_{B C S} / \zeta_{\omega \pm},
$$

where $\tilde{\omega}_{ \pm}=\omega+\epsilon_{b F} g_{B C S} \mp i J, \quad \zeta_{\omega \pm}=\sqrt{\widetilde{\omega}_{ \pm}^{2}-\left(\epsilon_{b F} f_{B C S}\right)^{2}}$, $\epsilon_{b F}=D_{F} /\left(2 \gamma_{F} d_{F}\right)$. One can see that in the limiting cases of small and large energy $\epsilon_{b F}$ the functions $g_{F \pm}, f_{F \pm}$ describe a superconducting state with the energy gap equal to $\epsilon_{b F}$ if $\epsilon_{b F} \ll \Delta$ (a subgap in the excitation spectrum) and to $\Delta$ in the opposite case. In both cases the position of the energy gap is shifted with respect to $\epsilon=0$ (the Matsubara frequencies are related to $\epsilon$ via $\omega=-i \epsilon$ ). It can be easily shown that the function $g_{F 3}$ that determines the magnetization, Eqs. (2) and (17), equals zero for $\epsilon_{b F}=0$ (very small S/F interface transparency) and for very large values of $\epsilon_{b F}$ (a perfect S/F contact). This dependence of $\delta M_{F}$ on $R_{b}$ leads to a nonmonotonic behavior of the change of the magnetization $\delta M_{F, S}$. In Fig. 2 we show the temperature dependence of $\delta M_{F, S}(0)$ for values of the parameters similar to those of Ref. 14. We see that the decrease of the magnetization may be of the order of $10 \%$ and larger. This result correlates with the experimental data of Ref. 14 (see Ref. 21). We have checked that $\left|f_{S 0}\right|<1$ for the parameters in Fig. 2.

We also present here analytical formulas for the ratio $r_{S, F}=\delta M_{S, F}(0) / M_{F 0}$ using Eq. (18) and considering the case of low temperatures $(T \ll \Delta) ; \delta M_{S, F}$ are the magnetization variations in the $\mathrm{S}$ and $\mathrm{F}$ films and $M_{F 0}$ is the magnetization in the F film above $T_{c}$. The relation between $M_{F 0}$ and $J$ depends on a particular model of the ferromagnet. For ex-

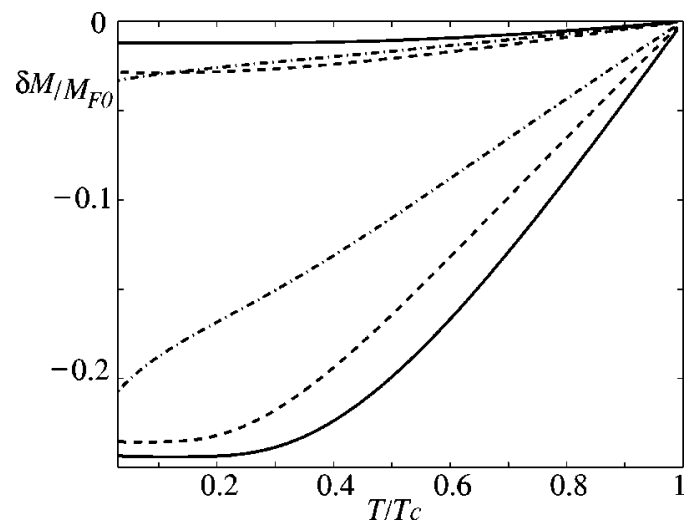

FIG. 2. Temperature dependence of $\delta M_{S}(0) / M_{F 0}$ (lower curves) and $\delta M_{F}(0) / M_{F 0}$ (upper curves) for the following values of $\bar{\gamma}_{F}=\gamma_{F} / \xi_{0} \quad\left(\xi_{0}=\sqrt{D_{S} / 2 T_{c}}\right): \quad \bar{\gamma}_{F}=0.1 \quad$ (solid line), $\quad \bar{\gamma}_{F}=0.3$ (dashed line), and $\bar{\gamma}_{F}=0.5$ (dot-dashed line). Here $\gamma_{F} / \gamma_{S}=0.5$, $J / T_{c}=20$ and $d_{F} / \xi_{0}=0.1$.

ample, in the simplest model of the ferromagnet with a constant and positive $J$ we have for an itinerant ferromagnet $M_{F 0}=g \mu_{B} \nu J$ (see Ref. 21). To simplify the expressions for $r_{S, F}$ we assume also that $J \ll \epsilon_{b F} \approx\left(D_{F} / d_{F}^{2}\right)\left(R_{F} / R_{b}\right)$ $\times\left(d_{F} / \xi_{F}\right)$ (this limit may correspond to the experiment ${ }^{14}$ ). In this case we obtain

$$
r_{S} \approx-1.67 \sqrt{\Delta d_{F}^{2} / D_{S}},
$$

$$
r_{F} \approx-\pi \Delta / 2 \epsilon_{b F}
$$

For estimations of the parameters one can take experimental values from Ref. 14 where a "weak" ferromagnet $\mathrm{Pd}_{(1-x)} \mathrm{Fe}_{x}$ was used. One gets $D_{F} / d_{F}^{2}=1000 \mathrm{~K}$ for $d_{F}=20 \mathrm{~A}$. The $\mathrm{Cu}$ rie temperature which may be of the order of $J$ varied from 90 to $250 \mathrm{~K}$. The barrier (interface) resistance $R_{b}$ is not known, but one can give a crude estimation noting that $\left(R_{F} / R_{b}\right)\left(d_{F} / \xi_{F}\right) \approx T_{t r}$, where $T_{t r}$ is the transmission coefficient which varies from very small values to a value of the order 1.

In conclusion, we have demonstrated the existence of the inverse proximity effect in $\mathrm{S} / \mathrm{F}$ structures. Due to the presence of the superconductor the magnetization in the ferromagnet with the ferromagnetic type of the exchange interaction is reduced and a magnetic moment is induced in the superconductor below $T_{c}$. Its direction is opposite to the direction of the magnetic moment in the ferromagnet and spreads over the superconducting coherence length $\xi_{S}$. This distance can be much larger than the F film thickness. The effect discussed may be the reason for a reduced magnetization observed in S/F structures leading to a frequency shift of the magnetic resonance. ${ }^{14}$ This conclusion is changed in the case of ferromagnets with the antiferromagnetic interaction between free electrons and localized moments [negative $J_{1}$ in Eq. (8)]. If a contribution of localized moments to the total magnetization in such ferromagnets dominates $\left(M_{l o c}\right.$ 
$>M_{e}$ ), the magnetization $\mathbf{M}_{e}$ is opposite to $\mathbf{M}$ and therefore the induced magnetization variation in the superconductor $\delta M_{S}$ will be parallel to $\mathbf{M}$.

We recently became aware of the paper Ref. 22 where the magnetization leakage into the $\mathrm{S}$ layer was numerically cal- culated for a ballistic S/F structure. In this case the magnetization penetrates the $\mathrm{S}$ layer over distances of the order of the Fermi wavelength. We are not interested in small scales of this order.

We would like to thank SFB 491 for financial support.
${ }^{1}$ P.G. de Gennes, Rev. Mod. Phys. 36, 225 (1964).

${ }^{2}$ A.I. Buzdin, L.N. Bulaevskii, and S.V. Panyukov, JETP Lett. 35, 178 (1982).

${ }^{3}$ I. Baladie and A. Buzdin, Phys. Rev. B 64, 224514 (2001).

${ }^{4}$ F.S. Bergeret, A.F. Volkov, and K.B. Efetov, Phys. Rev. B 65, 134505 (2002).

${ }^{5}$ F.S. Bergeret, A.F. Volkov, and K.B. Efetov, Phys. Rev. Lett. 86, 4096 (2001); 90, 117006 (2003).

${ }^{6}$ A. Kadigrobov, R.I. Shekhter, and M. Jonson, Europhys. Lett. 54, 394 (2001).

${ }^{7}$ Z. Radovic, Marko Ledvij, Ljiljana Dobrosavljevic-Grujic, A.I. Buzdin, and John R. Clem, Phys. Rev. B 44, 759 (1991).

${ }^{8}$ E.A. Demler, G.B. Arnold, and M.R. Beasley, Phys. Rev. B 55, 15 174 (1997).

${ }^{9}$ K. Halterman and O.T. Valls, Phys. Rev. B 65, 014509 (2002); 66, 224516 (2002).

${ }^{10}$ Yu.A. Izyumov, Yu N. Proshin, and M.G. Khusainov, Usp. Fiz. Nauk 172, 113 (2002).

${ }^{11}$ R. Fazio and C. Lucheroni, Europhys. Lett. 45, 707 (1999).

${ }^{12}$ V.N. Krivoruchko and E.A. Koshina, Phys. Rev. B 66, 014521 (2002).

${ }^{13}$ Th. Muehge, N.N. Garif'yanov, Yu. V. Goryunov, K. Theis-Bröhl, K. Westerholt, I.A. Garifullin, and H. Zabel, Physica C 296, 325
(1998).

${ }^{14}$ I.A. Garifullin, D.A. Tikhonov, N.N. Garif'yanov, M.Z. Fattakhov, K. Theis-Bröhl, K. Westerholt, and H. Zabel, Appl. Magn. Reson. 22, 439 (2002).

${ }^{15}$ F.S. Bergeret, A.F. Volkov, and K.B. Efetov, Phys. Rev. B 68, 064513 (2003).

${ }^{16}$ L.P. Gor'kov and A.L. Rusinov, Sov. Phys. JETP 19, 922 (1964).

${ }^{17}$ C.A. Balseiro and L.M. Falicov, Phys. Rev. B 19, 2548 (1979).

${ }^{18}$ A. I. Larkin and Yu. N. Ovchinnikov, in Nonequilibrium Superconductivity, edited by D.N. Langenberg and A.I. Larkin (Elsevier, Amsterdam, 1984).

${ }^{19}$ F.S. Bergeret, K.B. Efetov, and A.I. Larkin, Phys. Rev. B 62, 11 872 (2000).

${ }^{20}$ A.I. Buzdin and L.N. Bulaevskii, Sov. Phys. JETP 67, 576 (1988).

${ }^{21}$ Note that we normalized $\delta M$ with respect to the magnetization $M$ of the ferromagnet above $T_{c}$ assuming that $M$ is determined by the free electrons (itinerant ferromagnets). In the case of ferromagnets with localized magnetic moments and the ferromagnetic type of interaction all functions $\delta M / M$ should be multiplied by a factor $(1+\lambda)^{-1}$, where $\lambda=M_{l o c} / M$ and $M_{l o c}$ is the contribution to the magnetization due to localized moments.

${ }^{22}$ K. Halterman and O.T. Valls, Phys. Rev. B 69, 014517 (2004). 